\title{
The morphologic spectrum and clinical significance of light chain proximal tubulopathy with and without crystal formation
}

\author{
Christopher P Larsen, Jane M Bell, Alexis A Harris, Nidia C Messias, Yihan H Wang and \\ Patrick D Walker
}

Nephropathology Associates, Little Rock, AR, USA

\begin{abstract}
The renal diseases most frequently associated with myeloma include amyloidosis, monoclonal immunoglobulin deposition disease, and cast nephropathy. Less frequently reported is light chain proximal tubulopathy, a disease characterized by $\kappa$-restricted crystal deposits in the proximal tubule cytoplasm. Light chain proximal tubulopathy without crystal deposition is only loosely related to the typical light chain proximal tubulopathy, and little is known about this entity. A search was performed of the 10081 native kidney biopsy samples processed by our laboratory over the past 2 years for cases that had light chain restriction limited to the proximal tubule cytoplasm. A total of 10 cases of light chain proximal tubulopathy without crystal deposition were found representing $3.1 \%$ of light chain-related diseases. Nine of these 10 showed $\lambda$-light chain restriction. Only three cases of light chain proximal tubulopathy with crystals were found accounting for $0.9 \%$ of light chain-related diseases. Two of these three were $\kappa$ subtype. Plasma cell dyscrasia was unsuspected in seven of the 10 patients with light chain proximal tubulopathy without crystals at the time of renal biopsy. After the biopsy was reported, follow-up was available on 9/10 patients with 9/9 showing a plasma cell dyscrasia including 8/9 with multiple myeloma. We found that light chain proximal tubulopathy without crystal formation, despite being rarely described in the literature, is over three times more common than light chain proximal tubulopathy with crystal formation in our series. And given that it is often associated with previously unrecognized myeloma, it is a critically important diagnosis.

Modern Pathology (2011) 24, 1462-1469; doi:10.1038/modpathol.2011.104; published online 24 June 2011
\end{abstract}

Keywords: kidney; light chain proximal tubulopathy; multiple myeloma; plasma cell dyscrasia; renal biopsy

Kidney disease is a common manifestation of multiple myeloma with almost one half of all patients showing some degree of renal failure at presentation. Renal involvement is a poor prognostic indicator and is the second leading cause of death in myeloma after infection. ${ }^{1-3}$ The characteristic renal abnormalities in myeloma result from the production of a paraprotein by the clonal plasma cells. The most common of these paraproteinemicrelated renal diseases include amyloidosis, light chain cast nephropathy, and light chain deposition disease.

Correspondence: Dr CP Larsen, MD, Nephropathology Associates, 10810 Executive Center Drive, Suite 100, Little Rock, AR 72211, USA.

E-mail: Chris.larsen@nephropath.com

Received 18 December 2010; revised 11 May 2011; accepted 12 May 2011; published online 24 June 2011
Light chain proximal tubulopathy is an uncommonly reported paraproteinemic-related renal disease described in case reports or small case series. The classic description is the presence of $\kappa$-restricted crystal deposits in the proximal tubular cytoplasm. These patients frequently present with Fanconi syndrome in the setting of smoldering myeloma. ${ }^{4}$ Occasionally, cases are seen with light chain restriction in the proximal tubules that have no evidence of crystal formation in the proximal tubular cytoplasm. These cases of light chain proximal tubulopathy without crystal deposition are only loosely related to the typical light chain proximal tubulopathy. Little is known about this entity as only three cases have previously been reported $^{5}$ compared with over 50 cases of light chain proximal tubulopathy with crystals. The aim of this study was to determine the relative frequency and significance of kidney biopsies involved by isolated light chain proximal tubulopathy without 
intra-cytoplasmic crystals in a single large referral center.

\section{Materials and methods}

We searched the biopsy files of our laboratory going back 2 years for cases with evidence of light chain restriction within the proximal tubular cytoplasm with no other evidence of paraproteinemic-related renal disease. Specifically, cases were excluded that showed evidence of concurrent amyloidosis, light chain deposition disease, and light chain cast nephropathy. All cases were processed by light, immunofluorescence, and electron microscopy using routine techniques briefly described below.

\section{Light Microscopy}

Briefly, kidney biopsy samples were fixed in buffered formalin, dehydrated in graded alcohols, and embedded in paraffin using standard techniques. Serial 3- $\mu \mathrm{m}$ thick sections were cut and treated with hematoxylin and eosin, Jones methenamine silver, Masson trichrome, and periodic acid-Schiff reagent. In addition, congo red staining was performed in each case on $5-\mu \mathrm{m}$ thick sections.

\section{Immunofluorescence Microscopy}

Samples were transported in Michel's media, washed in buffer, and frozen in a cryostat. Sections, cut at $5 \mu \mathrm{m}$, were rinsed in buffer and reacted with fluorescein-tagged polyclonal rabbit anti-human antibodies to IgG, IgA, IgM, C3, C4, C1q, fibrinogen, and $\kappa$-, and $\lambda$-light chains (all from Dako, Carpenteria, CA, USA) for $1 \mathrm{~h}$, rinsed, and a coverslip applied using aqueous mounting media.

\section{Electron Microscopy}

The ends of the renal biopsy specimen were removed as $1 \mathrm{~mm}$ cubes, dehydrated using graded alcohols and embedded in epon/araldite resin. Sections $1-\mu \mathrm{m}$ thick were cut using an ultramicrotome, stained with toluidine blue and examined with a light microscope. Thin sections were examined in a Jeol JEM-1011 electron microscope (Jeol, Tokyo, Japan). Photomicrographs were routinely taken at $\times 5000, \times 12000$, and $\times 20000$ magnifications.

The morphological features were detailed in each case by four renal pathologists (AH, CL, NM, and YW). The pathologists were blinded to the patient's clinical history at the time of evaluation. Special emphasis was given to the proximal tubular findings during this evaluation.

\section{Results}

Among the 10081 native kidney biopsies processed in our laboratory over the previous 2-year period, there were $322(3.2 \%)$ with evidence of a paraproteinemic-related disease (Table 1). Among these were 13 cases with isolated light chain proximal tubulopathy including 10 with no evidence of intracytoplasmic crystals and three with intracytoplasmic crystal formation. As per the inclusion criteria, the light chain restriction on immunofluorescence microscopy was limited to the proximal tubular cytoplasm in each case.

The clinical findings of the 13 patients with light chain proximal tubulopathy are summarized in Table 2. The indication for biopsy in patients with light chain proximal tubulopathy without crystals was isolated proteinuria $(4 / 10)$ and progressive renal insufficiency with proteinuria (6/10). Only three patients had a known plasma cell dyscrasia at the time of biopsy. But all patients were found to have a free-light chain in their urine and/or serum, which matched with that seen in the proximal tubular cytoplasm after the biopsy results were known. There were a total of nine patients, in which the biopsy showed light chain proximal tubulopathy without crystals who were available for follow-up. Among these patients, 9/9 had or were found to have a plasma cell dyscrasia with $8 / 9$ showing multiple myeloma. Five of the nine patients with light chain proximal tubulopathy without crystal formation were found to have no evidence of Fanconi syndrome, and the remaining four were not evaluated for the presence of a Fanconi syndrome.

The biopsies had from 11 to 42 glomeruli per sample with an average of 25 . The proximal tubular findings by light and electron microscopy are detailed in Table 3 . Seven of the 10 biopsies that had light chain proximal tubulopathy without crystal formation had nonspecific evidence of acute tubular injury within the proximal tubules (detailed in Table 3 and Figure 1). In the remaining three cases, there were no significant changes within the proximal tubules by light and electron microscopy. The immunofluorescence findings were limited to the presence of light chain restriction in the cytoplasm of tubules located only in the renal cortex

Table 1 Frequency of light chain-related diseases in 322 renal biopsies

\begin{tabular}{lrrrr}
\hline Disease & $\kappa$ & $\lambda$ & Total & Total \% \\
\hline LCDD & 38 & 11 & 49 & 15.2 \\
LCCN & 17 & 27 & 44 & 13.7 \\
LCDD+LCCN & 35 & 20 & 55 & 17.1 \\
Amyloid & 27 & 130 & 157 & 48.8 \\
Amyloid +LCDD+LCCN & 1 & 3 & 4 & 1.2 \\
LCPT & 3 & 10 & 13 & 4.0 \\
\hline
\end{tabular}

Abbreviations: LCDD, light chain deposition disease; LCCN, light chain cast nephropathy; LCPT, light chain proximal tubulopathy. 
Table 2 Clinical findings in patients with light chain proximal tubulopathy

\begin{tabular}{|c|c|c|c|c|c|c|c|}
\hline $\begin{array}{l}\text { Case } \\
\text { number }\end{array}$ & Crystals? & Age/sex & $\begin{array}{l}\text { Indication } \\
\text { for biopsy }\end{array}$ & $\begin{array}{c}C r \\
(m g / d l)\end{array}$ & $\begin{array}{c}\text { Prot } \\
(\mathrm{g} / 24 \mathrm{~h})\end{array}$ & $\begin{array}{l}\text { PCD diagnosis } \\
\text { before biopsy }\end{array}$ & $\begin{array}{l}\text { PCD diagnosis } \\
\text { after biopsy }\end{array}$ \\
\hline 1 & $\mathrm{~N}$ & $53 / \mathrm{F}$ & Prot & 0.9 & 3.5 & None & Myeloma \\
\hline 2 & $\mathrm{~N}$ & $68 / \mathrm{M}$ & Prot & 0.9 & 3 & None & Myeloma \\
\hline 3 & $\mathrm{~N}$ & $78 / \mathrm{F}$ & PRF & 2.1 & 0.5 & Plasmacytoma & Plasmacytoma \\
\hline 4 & $\mathrm{~N}$ & $53 / \mathrm{F}$ & NS and PRF & 1.8 & 4.3 & None & Myeloma \\
\hline 5 & $\mathrm{~N}$ & $78 / \mathrm{M}$ & Prot & Unk & 2 & Myeloma & Myeloma \\
\hline 6 & $\mathrm{~N}$ & $48 / \mathrm{F}$ & ARF & 10.2 & 12 & None & Myeloma \\
\hline 7 & $\mathrm{~N}$ & $78 / \mathrm{F}$ & PRF & 2.7 & 10 & Myeloma & Myeloma \\
\hline 8 & $\mathrm{~N}$ & $58 / \mathrm{M}$ & PRF & 2.1 & 1.2 & None & Smoldering myeloma \\
\hline 9 & $\mathrm{~N}$ & $71 / \mathrm{M}$ & PRF and Prot & 1.1 & 3.4 & None & Myeloma \\
\hline 10 & $\mathrm{~N}$ & $71 / \mathrm{M}$ & Prot & 1 & 0.6 & None & Lost to follow up \\
\hline 11 & $\mathrm{Y}$ & $81 / \mathrm{M}$ & PRF and Prot & 1.7 & 6.7 & None & Smoldering myeloma \\
\hline 12 & $\mathrm{Y}$ & $73 / \mathrm{F}$ & CKD V & 3.9 & Unk & None & Myeloma \\
\hline 13 & $\mathrm{Y}$ & $61 / F$ & CKD IV & 1.5 & Unk & Unk & Unk \\
\hline
\end{tabular}

Abbreviations: ARF, Acute renal failure; CKD, chronic kidney disease; PCD, plasma cell dyscrasia; Prot, proteinuria; PRF, progressive renal failure; Unk, unknown; NS, nephrotic syndrome.

Table 3 Morphological findings within proximal tubules

\begin{tabular}{|c|c|c|c|}
\hline $\begin{array}{l}\text { Case } \\
\text { number }\end{array}$ & Light microscopy & $I F$ & Electron microscopy \\
\hline 1 & WNL & $\lambda$ & Lysosomes slightly increased in number \\
\hline 2 & WNL & $\lambda$ & $\begin{array}{l}\text { Lysosomes increased in number including some with } \\
\text { mottled appearance and irregular membranes }\end{array}$ \\
\hline 3 & $\begin{array}{l}\text { Diffuse flattening with loss of brush border and focal } \\
\text { denudation }\end{array}$ & $\lambda$ & $\begin{array}{l}\text { Focal swollen mitochondria and mild increase in } \\
\text { lysosomes including some with mottled appearance }\end{array}$ \\
\hline 4 & $\begin{array}{l}\text { Diffuse apical cytoplasmic blebbing, focal flattening, } \\
\text { and focal dilatation }\end{array}$ & $\lambda$ & $\begin{array}{l}\text { Increased lysosomes including some with mottled } \\
\text { appearance and irregular membranes }\end{array}$ \\
\hline 5 & Diffuse apical cytoplasmic blebbing and focal dilatation & $\lambda$ & $\begin{array}{l}\text { Increased lysosomes including some with mottled } \\
\text { appearance and irregular membranes }\end{array}$ \\
\hline 6 & $\begin{array}{l}\text { Diffuse apical cytoplasmic blebbing, focal flattening, } \\
\text { and focal dilatation }\end{array}$ & $\lambda$ & Lysosomes increased in number \\
\hline 7 & Diffuse flattening with loss of brush border & $\lambda$ & $\begin{array}{l}\text { Focal mitochondrial swelling, increased lysosomes } \\
\text { including some with mottled appearance }\end{array}$ \\
\hline $8 a^{a}$ & Diffuse cytoplasmic swelling and apical blebbing & $\lambda$ & $\begin{array}{l}\text { Focal mitochondrial swelling, increased lysosomes } \\
\text { including some with mottled appearance }\end{array}$ \\
\hline $8 b^{\mathrm{a}}$ & Diffuse cytoplasmic swelling and apical blebbing & $\lambda$ & $\begin{array}{l}\text { Focal mitochondrial swelling, increased lysosomes } \\
\text { including some with mottled appearance }\end{array}$ \\
\hline 9 & Focal cytoplasmic swelling and apical blebbing & $\lambda$ & Focal mitochondrial swelling, increased lysosomes \\
\hline 10 & WNL & $\kappa$ & Lysosomes slightly increased in number \\
\hline 11 & $\begin{array}{l}\text { Diffuse cytoplasmic vacuolization and apical blebbing, } \\
\text { and rare intracytoplasmic crystals }\end{array}$ & $\lambda^{\mathrm{b}}$ & Rare intracytoplasmic crystals \\
\hline 12 & Cytoplasm packed with crystals & $\kappa$ & Numerous intracytoplasmic crystals \\
\hline 13 & Cytoplasm packed with crystals & $\kappa$ & Numerous intracytoplasmic crystals \\
\hline
\end{tabular}

Abbreviations: IF, immunofluorescence; NP, not performed; WNL, within normal limits.

${ }^{\mathrm{a}}$ Patient 8 had two biopsies.

${ }^{\mathrm{b}} \mathrm{IF}$ was performed on paraffin-embedded tissue following pronase digestion.

in all cases except patient 11, who had a concurrent membranous glomerulopathy, which showed the typical presence of granular IgG deposits along the glomerular basement membranes. The glomeruli were unremarkable in the remaining cases without evidence of either immunoglobulin deposition or foot process effacement. Patient 8 had a follow-up biopsy at 13 months after the original that showed almost identical findings without significant progression of disease. One of the cases with crystals did not show staining of the crystalline deposits after routine IF. However, it showed $\lambda$ restriction after pronase digestion on paraffin-embedded material by immunofluorescence.

\section{Discussion}

We present 13 new cases of light chain proximal tubulopathy including 10 cases without crystal formation and describe the morphological findings and clinical significance of this entity. Light chain 

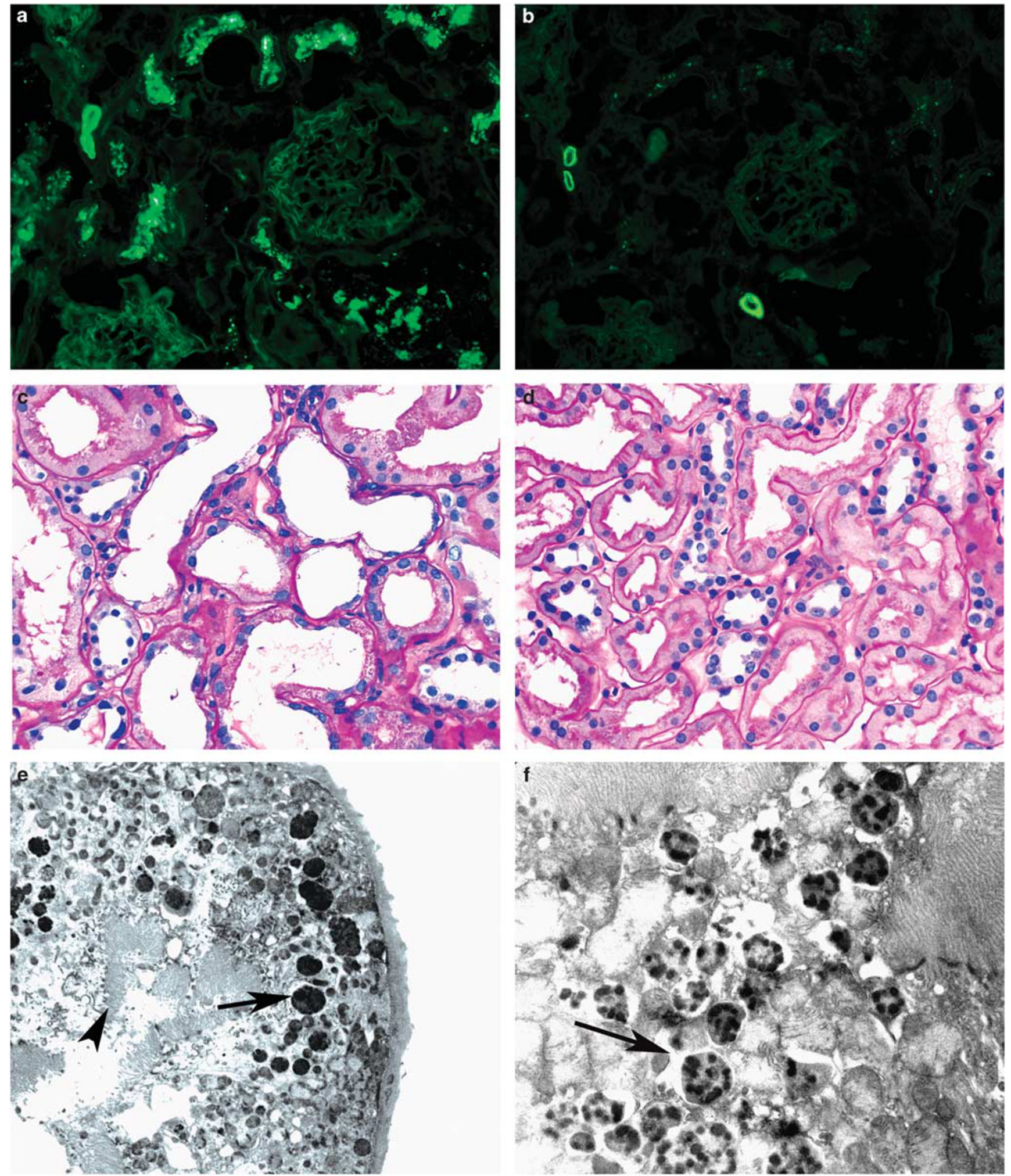

Figure 1 Light chain proximal tubulopathy without crystal formation. (a and b) Serial sections of the same field stained with $\lambda(\mathbf{a})$ and $\kappa$ (b) reveals $\lambda$ restriction in the proximal tubular cytoplasm (Direct immunofluorescence; original magnification $\times 100$ ). (c) Nonspecific acute tubular injury with tubular epithelium thinning, loss of brush border, and focal epithelial denudation (periodic acid-Schiff; original magnification $\times 400$ ). (d) Biopsy samples from patient with isolated proteinuria showing normal proximal tubules with intact brush borders (periodic acid-Schiff; original magnification $\times 400$ ). (e and $\mathbf{f}$ ) Transmission electron photomicrographs showing a proximal tubule with disruption of the brush border (arrowhead), increased lysosomes with irregular contours and a mottled appearance (arrow), and no evidence of crystal formation (unstained; original magnification $\times 12000$ and $\times 20000$ ). 
proximal tubulopathy without crystal formation was found to be over three times more prevalent in our series than the more commonly described light chain proximal tubulopathy with crystal formation. In our study, light chain proximal tubulopathy is the most likely cause of the renal dysfunction that led to the biopsy in each case. Regardless of the pathogenecity, it is clear that this morphological finding on renal biopsy is clinically significant. All patients available for follow-up had a plasma cell dyscrasia and the vast majority were subsequently found to have multiple myeloma despite the fact that only one third of them had a known history of plasma cell dyscrasia at the time of biopsy. This study underscores the importance of careful interpretation of the $\kappa$ and $\lambda$ immunofluorescence stains on renal biopsy, as subtle findings such as these, can have significant clinical impact.

The pathogenic nature of a given free-light chain is inherent to the light chain structure itself. Solomon $^{6}$ demonstrated that mice injected with human free-light chains developed renal lesions similar to those seen in the kidney biopsy samples of the patient they were taken from in most cases. ${ }^{6}$ Several unique biochemical properties of light chains from patients with light chain proximal tubulopathy with crystal formation have been demonstrated that explain the morphological lesions seen on biopsy. The variable domain of these light chains shows resistance to proteolysis by the tubular lysosomal enzymes pepsin and cathepsin B, whereas the light chains from patients with LCCN and control patients are fully digested by these enzymes. ${ }^{7}$ Additionally, this protease-resistant variable domain has been shown to spontaneously form crystals in vitro that are morphologically similar to those seen in the tubular cytoplasm. ${ }^{8}$ A logical sequence of events leading to the formation of crystals within the tubular cytoplasm can be summarized as follows. First, the free-light chains pass through the glomerular basement membranes and undergo endocytosis by the proximal tubular epithelial cells. Following endocytosis, the variable region of the light chain resists degradation and accumulates within the lysosome where it undergoes spontaneous crystallization. The lack of tubular casts could possibly be explained by the fact that these crystal-forming light chains, unlike free-light chains from most patients with LCCN, have a relatively low affinity for Tamm Horsfall protein,,$^{7,9,10}$ which results in a lower propensity to form casts in the distal tubules.

The $\lambda$ predominance seen in the cases of light chain proximal tubulopathy without crystal formation suggest that the light chains involved in this entity might also possess some unique characteristics leading to the findings seen on renal biopsy. It is possible that these light chains also possess resistance to degradation by lysosomal enzymes but lack the ability to spontaneously crystallize. This would explain the apparent build-up of light chains in the proximal tubule cells without crystal formation. The lack of casts in these cases suggests that the light chains have a relatively low affinity for Tamm Horsfall protein, and the absence of amyloidosis suggests that these light chains are not particularly amyloidogenic. Another possibility is that these findings represent the early stages of disease in which the intra-tubular and/or cytoplasmic crystals have not yet fully developed. The presence of a 13-month follow-up biopsy in one patient, which showed identical findings, makes this less likely. Additionally, the striking $\lambda$ predominance would be atypical for cases with crystal formation and/or casts.

The question also arises whether these light chains are pathogenic to the renal tubules or simply an incidental finding in patients with a plasma cell dyscrasia. Free-light chains are normally filtered through the glomerulus and the majority are reabsorbed by the proximal tubules where they undergo degradation within the lysosomes. ${ }^{11,12}$ Therefore, it is possible that the findings in this entity represent nothing more than a normal physiological process in a patient with excess free-light chains. Although we recognize this possibility we believe that, in most cases, the light chains are pathogenic to the tubules given the presence of an elevated serum creatinine in the majority of patients. Additionally, most of the biopsies showed morphological evidence of tubular injury without other significant predisposing factors. The suggestion that these light chains are pathogenic despite the lack of crystal formation is not without evidence in the literature, as the tubulopathic nature of free-light chains has been shown in multiple studies. One study examined the proximal tubular findings in patients with monotypic light chains compared with a group of patients with nonspecific proteinuria. ${ }^{13}$ None of the patients in this study showed evidence of crystal or cast formation. They found that the presence of monotypic light chains was associated with significantly increased cell damage and necrosis compared with patients without excess light chains in the urine. This same group found that human free-light chains and not albumin were toxic to rat proximal convoluted tubules. ${ }^{14}$ In vitro, free LCs and not human serum albumin were shown to lead to production of inflammatory cytokines in cultured human proximal tubule cells. ${ }^{12}$ Additionally, some patients with a plasma cell dyscrasia and clear evidence of proximal tubule injury leading to Fanconi syndrome show neither crystal formation in the proximal tubules nor casts in the distal tubules. ${ }^{4}$ These studies strongly suggest that the presence of excess free-light chains is sufficient to cause injury to renal proximal tubular cells whether or not crystals develop in the proximal tubular cytoplasm.

The findings in light chain proximal tubulopathy are frequently subtle in nature. In most cases, the clinical history available at the time of biopsy 

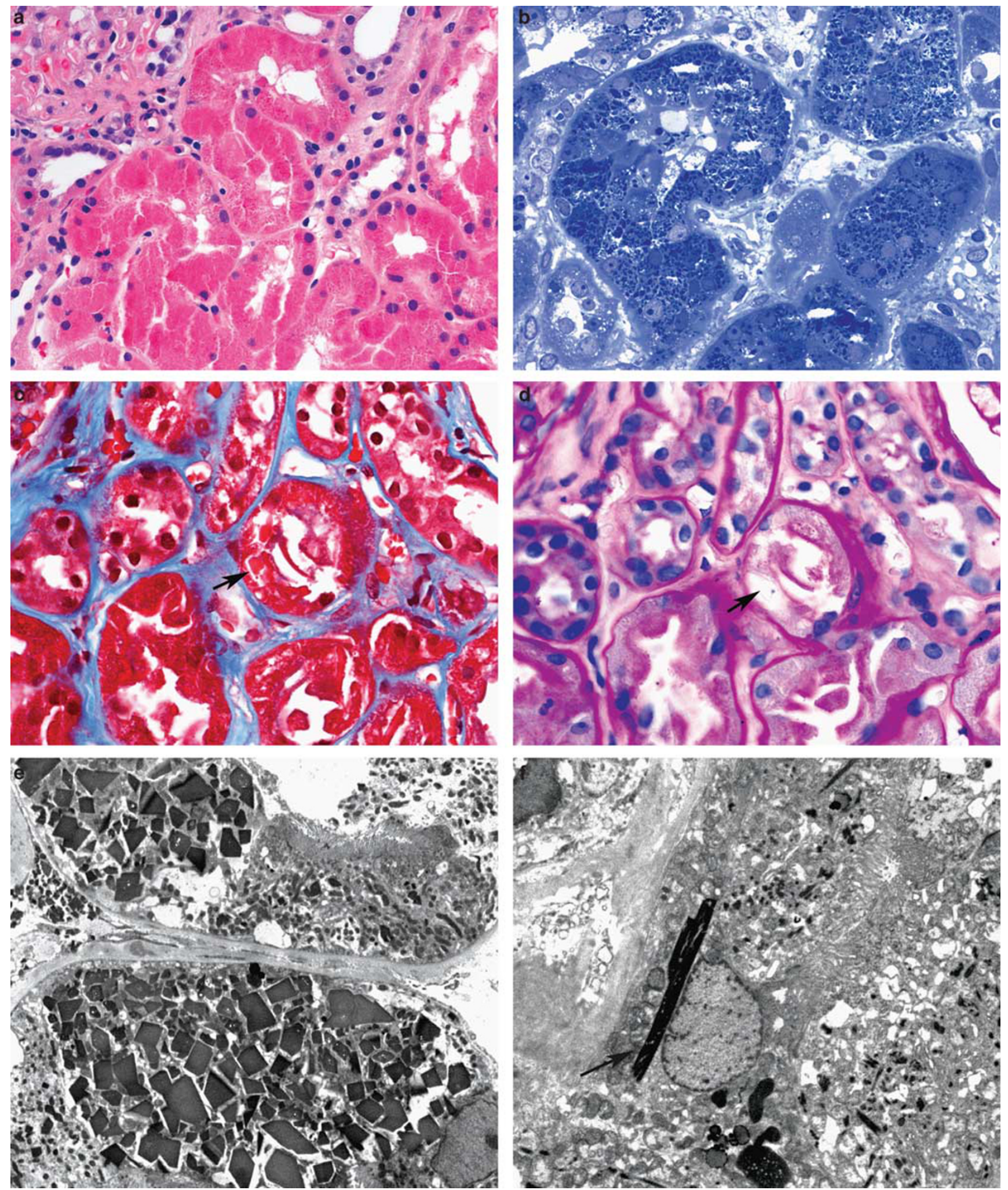

Figure 2 Light chain proximal tubulopathy with crystal formation. (a and b) Proximal tubules packed with brightly eosinophilic crystals on (a) hematoxylin and eosin stain (original magnification $\times 400$ ) which stain dark on (b) Toluidine blue (original magnification $\times 600$ ). (c and d) Images from case 11 show sparse crystal formation in the proximal tubules (arrows), which are brightly fuchsinophilic on (c) Masson trichrome stain (original magnification $\times 400$ ) and negative on periodic acid-Schiff (original magnification $\times 400$ ). (e and f) Transmission electron photomicrographs showing proximal tubules (e) packed with abundant rhomboid crystals in case 13 (unstained; original magnification $\times 8000$ ) and with (f) rare large rod-shaped crystals in case 11 (unstained; original magnification $\times 8000)$. 
will not include any information that heightens the index of suspicion for a paraproteinemic-related renal disease. If this proximal tubule light-chain restriction on immunofluorescence microscopy is not detected, it could delay the clinical diagnosis of the underlying plasma cell dyscrasia. This is true in cases with and without crystal formation. Although most cases of light chain proximal tubulopathy with crystals show florid intracytoplasmic deposition, some cases show very sparse crystal formation. In these cases we have found the trichrome and H\&E stains to be most useful for detecting the crystals on light microscopy as the crystals are brightly fuchsinophilic on trichrome and strongly eosinophilic on H\&E. The crystals are also easily seen on Toluidine blue stained EM thick sections. However, the crystals are negative by PAS (Figure 2). Compounding the difficulty in these cases is the fact that the crystals occasionally do not stain for $\kappa$ or $\lambda$ on routine immunofluorescence. Some have suggested the use of immunoelectron microscopy in these cases to increase the sensitivity of detecting light chain restriction. ${ }^{15}$ Although an elegant technique, we have found this to be unnecessary considering direct immunofluorescence performed on the paraffin-embedded tissue after pronase digestion, as well as routine immunoperoxidase, both reveal the light chain restriction in these cases. ${ }^{16-18}$ The most likely explanation for the increased sensitivity of these techniques over routine immunofluorescence lies in the addition of an antigen retrieval step. We found this to be true in one of the three cases of light chain proximal tubulopathy with crystals in this report (case 11). The cases without crystal formation can be even more subtle than those with crystals as there are no specific findings on light or electron microscopy to raise suspicion for a light chain-related disorder, and the only significant finding is the light chain restriction seen on immunofluorescence.

We are aware of a total of 56 cases with morphological evidence of light chain proximal tubulopathy reported in the literature. ${ }^{4-8,15,17-31}$ In these cases, 53/56 showed the presence of intracytoplasmic crystals in the proximal tubular cells and $37 / 40$ proved to be of the $\kappa$ subtype. The three previous cases without crystal formation were all reported by Kapur et $a l^{5}$ in one paper. All three cases were associated with a plasma cell dyscrasia that was unrecognized before the renal biopsy in two of three cases. All three were relatively unremarkable by light and electron microscopy and two of three cases were of the $\lambda$ subtype. Immunoelectron microscopy was performed in two of these cases and both showed light chain restriction within the lysosomes.

Kapur et $a l^{5}$ proposed maintaining the term light chain proximal tubulopathy for these cases without crystals rather than splitting them into a new diagnostic entity. We agree that these cases are best considered as a form of light chain proximal tubulopathy. However, the emphasis on crystal formation in this entity in the literature is difficult to explain, given the fact that light chain proximal tubulopathy without crystals is over three times more common in our material. This leads us to believe that cases without crystal formation are under-recognized. A missed diagnosis of light chain proximal tubulopathy without crystals would be a considerable error given the significance of the entity in our study.

\section{Disclosure/conflict of interest}

The authors declare no conflict of interest.

\section{References}

1 DeFronzo RA, Humphrey RL, Wright JR, et al. Acute renal failure in multiple myeloma. Medicine 1975; 54:209-223.

2 DeFronzo RA, Cooke CR, Wright JR, et al. Renal function in patients with multiple myeloma. Medicine 1978;57:151-166.

3 Korbet SM, Schwartz MM. Multiple myeloma. J Am Soc Nephrol 2006;17:2533-2545.

4 Messiaen T, Deret S, Mougenot B, et al. Adult Fanconi syndrome secondary to light chain gammopathy: clinicopathologic heterogeneity and unusual features in 11 patients. Medicine 2000;79:135-154.

5 Kapur U, Barton K, Fresco R, et al. Expanding the pathologic spectrum of immunoglobulin light chain proximal tubulopathy. Arch Pathol Lab Med 2007; 131:1368-1372.

6 Solomon A, Weiss DT, Kattine AA. Nephrotoxic potential of Bence Jones proteins. N Engl J Med 1991;324:1845-1851.

7 Leboulleux M, Lelongt B, Mougenot B, et al. Protease resistance and binding of Ig light chains in myelomaassociated tubulopathies. Kidney Int 1995;48:72-79.

8 Aucouturier $\mathrm{P}$, Bauwens $\mathrm{M}$, Khamlichi AA, et al. Monoclonal Ig L chain and L chain V domain fragment crystallization in myeloma-associated Fanconi's syndrome. J Immunol 1993;150:3561-3568.

9 Sanders PW, Booker BB, Bishop JB, et al. Mechanism of intranephronal proteinaceous cast formation by low molecular weight proteins. J Clin Invest 1990;85:570-576.

10 Huang ZQ, Sanders PW. Localization of a single binding site for immunoglobulin light chains on human Tamm-Horsfall glycoprotein. J Clin Invest 1997;99:732-736.

11 Maack T, Johnson V, Kau ST, et al. Renal filtration, transport, and metabolism of low-molecular-weight proteins: a review. Kidney Int 1979;16:251-270.

12 Sengul S, Zwinzinski C, Simon EE, et al. Endocytosis of light chains induces cytokines throgh activation of the NF- $\kappa \mathrm{B}$ in human proximal tubule cells. Kidney Int 2002;62:1977-1988.

13 Sanders PW, Herrera GA, Lott RL, et al. Morphologic alterations of the proximal tubules in light chainrelated renal disease. Kidney Int 1988;33:881-889.

14 Sanders PW, Herrera GA, Galla JH, et al. Human Bence Jones protein toxicity in rat proximal tubule epithelium in vivo. Kidney Int 1987;32:851-861. 
$15 \mathrm{Gu} \mathrm{X}$, Barrios R, Cartwright J, et al. Light chain crystal deposition as a manifestation of plasma cell dyscrasias: the role of immunoelectron microscopy. Hum Pathol 2003;34:270-277.

16 Nasr SH, Galgano SJ, Markowitz GS, et al. Immunofluorescence on pronase-digested paraffin sections: a valuable salvage technique for renal biopsies. Kidney Int 2006;70:2148-2151.

17 Herlitz LC, Roglieri J, Resta R, et al. Light chain proximal tubulopathy. Kidney Int 2009;76:792-797.

18 Yong JLC, Killingsworth MC. Acute light chain tubulopathy in myeloma. Pathology 2009;41:300-302.

19 Said SM, Assaad AM, Cerda J, et al. Light chain tubulopathy without Fanconi syndrome. Nephrol Dial Transplant 2006;21:3589-3590.

20 Elliott MR, Cortese C, Moreno-Aspitia A, et al. Plasma cell dyscrasia causing light chain tubulopathy without Fanconi syndrome. Am J Kid Dis 2010;55:1136-1141.

21 Maldonado JE, Velosa JA, Kyle RA, et al. Fanconi syndrome in adults. A manifestation of a latent form of myeloma. Am J Med 1975;58:354-364.

22 Thorner PS, Bédard YC, Fernandes BJ. Lambdalight-chain nephropathy with Fanconi's syndrome. Arch Pathol Lab Med 1983;107:654-657.

23 Cai G, Sidhu GS, Wieczorek R, et al. Plasma cell dyscrasia with kappa light-chain cyrstals in proximal tubular cells: a histological, immunofluorescent, and ultrastructural study. Ultrastruct Pathol 2006;30: 315-319.
24 Herrera GA, Sanders PW, Reddy BV, et al. Ultrastructural immunolabeling: a unique diagnostic tool in monoclonal light chain-related renal diseases. Ultrastruct Pathol 1994;18:401-416.

25 Markowitz GS, Flis RS, Kambham N, et al. Fanconi syndrome with free kappa light chains in the urine. Am J Kidney Dis 2000;35:777-781.

26 Truong LD, Mawad J, Cagle P, et al. Cytoplasmic crystals in multiple myeloma-associated Fanconi's syndrome. Arch Pathol Lab Med 1989;113:781-785.

27 Lajoie G, Leung R, Bargman JM. Clinical, biochemical, and pathological features in a patient with plasma cell dyscrasia and Fanconi syndrome. Ultrastruct Pathol 2000;24:221-226.

28 Pirani CL, Silva F, D’Agati V, et al. Renal lesions in plasma cell dyscrasias: ultrastructural observations. Am J Kid Dis 1987;10:208-221.

$29 \mathrm{Ma}$ CX, Lacy MQ, Rompala JF, et al. Acquired Fanconi syndrome is an indolent disorder in the absence of overt multiple myeloma. Blood 2004; 104:40-42.

30 Stokes MB, Aronoff B, Siegel D, et al. Dysproteinemiarelated nephropathy associated with crystal-storing histiocytosis. Kidney Int 2006;70:597-602.

31 Déret S, Denoroy L, Lamarine M, et al. Kappa light chain-associated Fanconi's syndrome: molecular analysis of monoclonal immunoglobulin light chains from patients with and without intracellular crystals. Protein Eng 1999;12:363-369. 